\title{
Traços de temperamento associados ao transtorno afetivo bipolar: uma revisão integrativa da literatura
}

\author{
Temperament traits associated with bipolar affective disorder: \\ an integrative literature review
}

\begin{abstract}
Alina Gomide Vasconcelos ${ }^{1}$, Leandro Fernandes Malloy-Diniz ${ }^{2}$, Elizabeth do Nascimento ${ }^{3}$, Fernando Neves ${ }^{4}$, Humberto Corrêa ${ }^{4}$
\end{abstract}

\begin{abstract}
Resumo
Estudos associam características do temperamento ao mau ajustamento e desenvolvimento de transtornos psiquiátricos, entendendo-os como aspectos de vulnerabilidade à psicopatologia. O objetivo deste estudo é realizar uma revisão integrativa da literatura a respeito dos traços de temperamento que caracterizam o transtorno afetivo bipolar em relação à população geral. Para tanto, foi realizada uma pesquisa sistemática sobre o tema nas bases de dados MEDLINE, PsycINFO e LILACS, utilizando os descritores bipolar disorder, temperament e/ou personality, no período compreendido entre janeiro de 2000 e dezembro de 2010. A consulta às bases de dados foi realizada em janeiro de 2011. A busca resultou em 199 artigos potenciais para compor a revisão. Após aplicação dos critérios de exclusão, 15 artigos foram selecionados para revisão e analisados na íntegra. Dentre os estudos selecionados, notou-se heterogeneidade no perfil das amostras e nos traços específicos de temperamento avaliados e respectivos instrumentos utilizados. Características de temperamento de pacientes bipolares são identificadas com base em diferentes modelos teóricos. De forma congruente, os resultados de cinco estudos apontaram que pacientes bipolares apresentam características relacionadas ao traço de personalidade denominado neuroticismo. Revisões futuras devem utilizar descritores mais específicos e restringir a busca a estudos com delineamento longitudinal.

Descritores: Temperamento, transtorno bipolar, revisão.
\end{abstract}

\begin{abstract}
Studies have suggested an association between temperament characteristics and adjustment and psychiatric disorders, describing them as different manifestations of vulnerability to psychopathology. The objective of this study was to conduct an integrative review of the literature on temperament traits typical of bipolar patients in relation to the general population. A systematic search was conducted on the MEDLINE, PsycINFO and LILACS databases, using the headings bipolar disorder, temperament and/or personality, between January 2000 and December 2010. The search was performed in January 2011. A total of 199 articles were identified for potential inclusion in the review. After application of the exclusion criteria, a total of 15 articles were selected and their full texts analyzed. Review of the selected studies revealed heterogeneity in terms of sample profile and specific temperament traits assessed with the appropriate instruments. Temperament traits in bipolar patients are identified based on different theoretical models. The results of five studies consistently showed that neuroticism is a distinct personality trait in the temperament profile of bipolar patients. Future reviews should use more specific keywords and limit the search to studies with a longitudinal design.
\end{abstract}

Keywords: Temperament, bipolar disorder, review.

\footnotetext{
1 Programa de Pós-Graduação em Neurociências, Universidade Federal de Minas Gerais (UFMG), Belo Horizonte, MG. ${ }^{2}$ Programa de Pós-Graduação em Neurociências, UFMG. Instituto Nacional de Ciência e Tecnologia de Medicina Molecular (INCT-MM). Laboratórios Integrados de Neuropsicologia (LINEU). ${ }^{3}$ Programa de Pós-Graduação em Psicologia, UFMG. Laboratório de Avaliação das Diferenças Individuais (LADI), UFMG. ${ }^{4}$ Programa de Pós-Graduação em Neurociências, UFMG. INCT-MM.

Recebido em 27/01/2011. Aceito em 16/06/2011. Não foram declarados conflitos de interesse associados à publicação deste artigo.

Como citar: Vasconcelos AG, Malloy-Diniz LF, Nascimento E, Neves F, Corrêa H. Traços de temperamento associados ao transtorno afetivo bipolar: uma revisão integrativa da literatura. Trends Psychiatry Psychother. 2011;33(3):169-80.
} 


\section{Introdução}

Os traços de temperamento são padrões emocionais reacionais inatos determinados por mecanismos de origem biológica. Tendem a ser disposições estáveis, no entanto estão sujeitos a mudanças causadas pela maturação e pela interação indivíduo-ambiente ao longo do desenvolvimento ${ }^{1}$. Apesar das inúmeras propostas teóricas, pode-se afirmar que, em linhas gerais, os padrões emocionais de respostas se referem às dimensões de reatividade (nível de atividade e emocionalidade) e autorregulação (atenção, abordagem e inibição) ${ }^{2}$. Um debate presente na literatura referente ao temperamento diz respeito aos limites desse construto em relação à personalidade, sendo que, algumas vezes, esses termos são utilizados de forma intercambiável. Na tentativa de esclarecer essa questão, Strelau ${ }^{1}$ propôs que a personalidade é um construto mais amplo que o temperamento, pois abarca outras características que são geralmente influenciadas pelo ambiente social ou pelos estágios de desenvolvimento do indivíduo, e envolveria ainda fenômenos como motivação, valores e interesses.

Dentre os modelos teóricos propostos para a investigação das dimensões da personalidade e do temperamento, a abordagem fatorial se destacou devido ao interesse em identificar os componentes da estrutura temperamental ${ }^{3}$. A literatura fundamentada nos modelos psicológicos fatoriais sugere que os aspectos temperamentais podem estar associados a diversos desfechos cotidianos, tais como desempenho no trabalho, saúde física, interesse musical, escolha do cônjuge, religiosidade, entre outros ${ }^{4}$. Há também estudos que associam características do temperamento na infância a transtornos psiquiátricos de forma geral ${ }^{5}$ e a transtornos de humor e ansiedade em particular na vida adulta, entendendo-os como aspectos de vulnerabilidade à psicopatologia6-9. Nesse sentido, os traços temperamentais poderiam representar manifestações endofenotípicas dos transtornos psiquiátricos ${ }^{10}$, e a identificação de um perfil temperamental pré-mórbido, uma fonte de informação útil para o desenvolvimento de intervenções precoces ${ }^{11,12}$. No final da década de 1970, foi proposto o conceito de temperamentos afetivos que inclui as dimensões de distimia, hipertimia, ciclotimia, irritável e ansioso ${ }^{13}$. A hipótese é de que a desregulação desses traços está associada à relação entre uma predisposição genético-familiar e os transtornos afetivos, tais como o transtorno afetivo bipo$\operatorname{lar}(\mathrm{TAB})^{13,14}$. Essa proposta sugere a presença de um continuum entre aspectos temperamentais e os transtornos afetivos. Logo, quanto mais extrema é a variação observada no temperamento, maior o risco de o indivíduo desenvolver o TAB.
Convergentes com essa visão, Hishfeld-Becker et al. ${ }^{15}$ propuseram que crianças com TAB apresentam características de temperamento pré-mórbidas relacionadas a dois construtos. O primeiro consiste na inibição-ativação comportamental frente a estímulos novos. Em casos extremos, indivíduos com alta ativação podem apresentar impulsividade e descontrole comportamental, facetas do temperamento relacionadas aos fatores de personalidade neuroticismo e extroversão, presentes nos modelos de Eysenck e dos cinco fatores ${ }^{15,16}$. Além disso, esse conceito se aproxima do termo "controle inibitório", definido na teoria de Rothbart et al. ${ }^{2}$ como descontrole comportamental, tal como proposto por Caspi \& Silva ${ }^{17}$, e também das dimensões temperamentais referentes à alta busca por novidades e baixa esquiva ao dano presentes no modelo psicobiológico de Cloninger et al. ${ }^{18}$. O segundo construto é a regulação emocional, que pode ser definida como o processo de iniciar, manter, modular e mudar a intensidade ou duração dos estados emocionais internos. Essa dimensão também pode ser associada à faceta impulsividade do fator neuroticismo do modelo dos cinco fatores ${ }^{15,16}$.

Em adultos bipolares, os modelos baseados nos sistemas motivacionais descritos por Jeffrey Gray, denominados sistema de ativação comportamental (behavioral activation system, BAS) e sistema de inibição comportamental (behavioral inhibition system, BIS), têm sido relacionados a diversas manifestações psicopatológicas ${ }^{19}$. Especificamente, Alloy et al. ${ }^{19}$ propõem que a desregulação do BAS pode influenciar o desencadeamento de sintomas maníacos e depressivos em indivíduos bipolares. De acordo com esses autores, uma hiperativação do BAS por eventos recompensadores estaria associada a emoções positivas (felicidade e elação, euforia, otimismo e excesso de autoconfiança), irritabilidade, baixa necessidade de sono e distraibilidade, o que predispõe ao desencadeamento de um episódio maníaco. Por outro lado, a desativação do BAS devido a eventos punitivos, tais como fracasso e falha ao alcançar objetivos, leva o indivíduo a apresentar tristeza, desesperança, baixa autoconfiança e baixo nível de energia, o que pode desencadear um episódio depressivo.

Pode-se observar que existem diferentes modelos teóricos subjacentes aos estudos que buscam caracterizar o perfil temperamental e associá-lo a variáveis clínicas de pacientes bipolares. Logo, o objetivo desta revisão foi identificar modelos teóricos e respectivos traços de temperamento associados a aspectos clínicos do TAB, com o intuito de contribuir para a compreensão da heterogeneidade das manifestações clínicas e comportamentais desse transtorno. Os objetivos específicos foram: 1) identificar modelos teóricos utilizados para fundamentar os estudos dos traços temperamentais em pacientes 
bipolares em relação à população em geral e os respectivos instrumentos objetivos utilizados para a avaliação dos mesmos; e 2) identificar traços de temperamento que podem ser aspectos protetores ou de vulnerabilidade ao TAB nas amostras investigadas.

\section{Método}

A pesquisa do material bibliográfico foi conduzida em quatro etapas. Na primeira etapa, foram definidas as bases de dados MEDLINE, PsycINFO e LILACS para a identificação dos artigos. A segunda etapa consistiu na definição dos descritores inseridos na busca dos artigos e dos critérios de inclusão. Os termos utilizados na busca foram delimitados a partir das palavras-chave presentes em artigos adequados ao tema, lidos previamente de forma não sistemática, e por meio de consulta às coleções de termos das respectivas bases de dados, a saber, Medical Subject Headings (MeSH) e Descritores em Ciências da Saúde (DeCS). Os descritores utilizados em conjunto para a identificação dos artigos foram: bipolar disorder (transtorno afetivo bipolar), temperament (temperamento) and/or (e/ou) personality (personalidade). A busca se restringiu a artigos publicados em português, inglês ou espanhol no período compreendido entre janeiro de 2000 e dezembro de 2010. A consulta às bases de dados foi realizada em janeiro de 2011 .

$\mathrm{Na}$ terceira etapa, realizou-se uma leitura dos títulos e resumos de todos os artigos selecionados a fim de identificar os trabalhos que abordavam o tema objeto da revisão, mesmo que de forma secundária, e ainda respeitavam os seguintes critérios de inclusão: 1) amostra clínica composta por bipolares ou, pelo menos, filhos de indivíduos bipolares e grupo controle composto por indivíduos sem histórico de diagnóstico de transtorno psiquiátrico; 2) avaliação dos traços de temperamento/ personalidade como variáveis independentes; 3) presença de medidas objetivas de investigação de traços de temperamento ou personalidade; 4) inserção de aspectos clínicos do TAB como variáveis dependentes do estudo. Foram excluídos estudos publicados sob a forma de editoriais, entrevistas, notas clínicas e revisões, como também aqueles que incluíram indivíduos vítimas de traumatismo cranioencefálico e doenças neurodegenerativas. Artigos que não atenderam a algum dos critérios propostos foram excluídos das análises posteriores.

A quarta etapa se referiu à análise sistemática dos artigos selecionados e à sua classificação em termos de nível de evidência com base nos critérios propostos por Manterola et al. ${ }^{20}$. A classificação proposta por esses autores possui cinco níveis de evidência, sendo que o nível I (mais alto) inclui os estudos meta-analíticos, e o nível mais baixo se refere a trabalhos em que há a opinião de especialistas sem avaliação clínica explícita (nível V). Essa classificação foi realizada no intuito de identificar a qualidade dos artigos selecionados para esta revisão.

\section{Resultados}

Foram encontrados 199 artigos potencialmente relevantes considerando a definição das bases de dados e os descritores (etapas 1 e 2). Na etapa 3, após a leitura dos títulos e resumos dos artigos, foram excluídos 153 estudos com base nos critérios apresentados, sendo que: 1) 35 eram estudos com amostras que incluíam participantes com características diversas daquelas delimitadas pela revisão, por exemplo, ausência de grupo controle e pacientes com transtorno desafiante opositor, disfunções neurológicas, entre outros; 2) 79 artigos não avaliaram traços de temperamento ou personalidade propriamente ditos e/ou não utilizaram medidas padronizadas, como, por exemplo, avaliação de estado de humor, criatividade, habilidades sociais, autoestima, entre outros; 3) 1 artigo utilizou método qualitativo para a coleta e análise dos dados; 4) 34 artigos não apresentavam dados originais (revisões narrativas, editoriais, comentários e notas clínicas); e 5) 4 artigos obtidos estavam duplicados em duas bases de dados. Na etapa 4, os 46 artigos selecionados como relevantes para o objetivo deste trabalho foram analisados na íntegra. A partir dessa análise, 31 artigos foram excluídos, sendo que: 1) 8 não apresentavam grupo controle formado por indivíduos sem histórico de diagnóstico de transtorno psiquiátrico; 2) 10 artigos não avaliaram traços de temperamento ou personalidade propriamente; 3) 12 artigos não apresentavam o TAB como variável dependente; e 4) 1 artigo não apresentou dados originais. Finalmente, a revisão foi realizada com base em 15 artigos. A Figura 1 apresenta esquematicamente o processo de levantamento bibliográfico e a seleção dos artigos dessa revisão.

Os resultados apresentados a seguir se restringem às análises conduzidas com o objetivo de identificar traços de temperamento característicos de pacientes bipolares quando comparados com indivíduos saudáveis.

\section{Síntese dos estudos}

Preliminarmente, serão apresentadas características das populações clínicas estudadas e os instrumentos utilizados para o diagnóstico de TAB e para a avaliação atual dos sintomas de humor. Em seguida, serão apresentados os modelos teóricos utilizados para fundamentar os estudos dos traços temperamentais no TAB e os respectivos instrumentos objetivos adotados para a avaliação dos mesmos. 


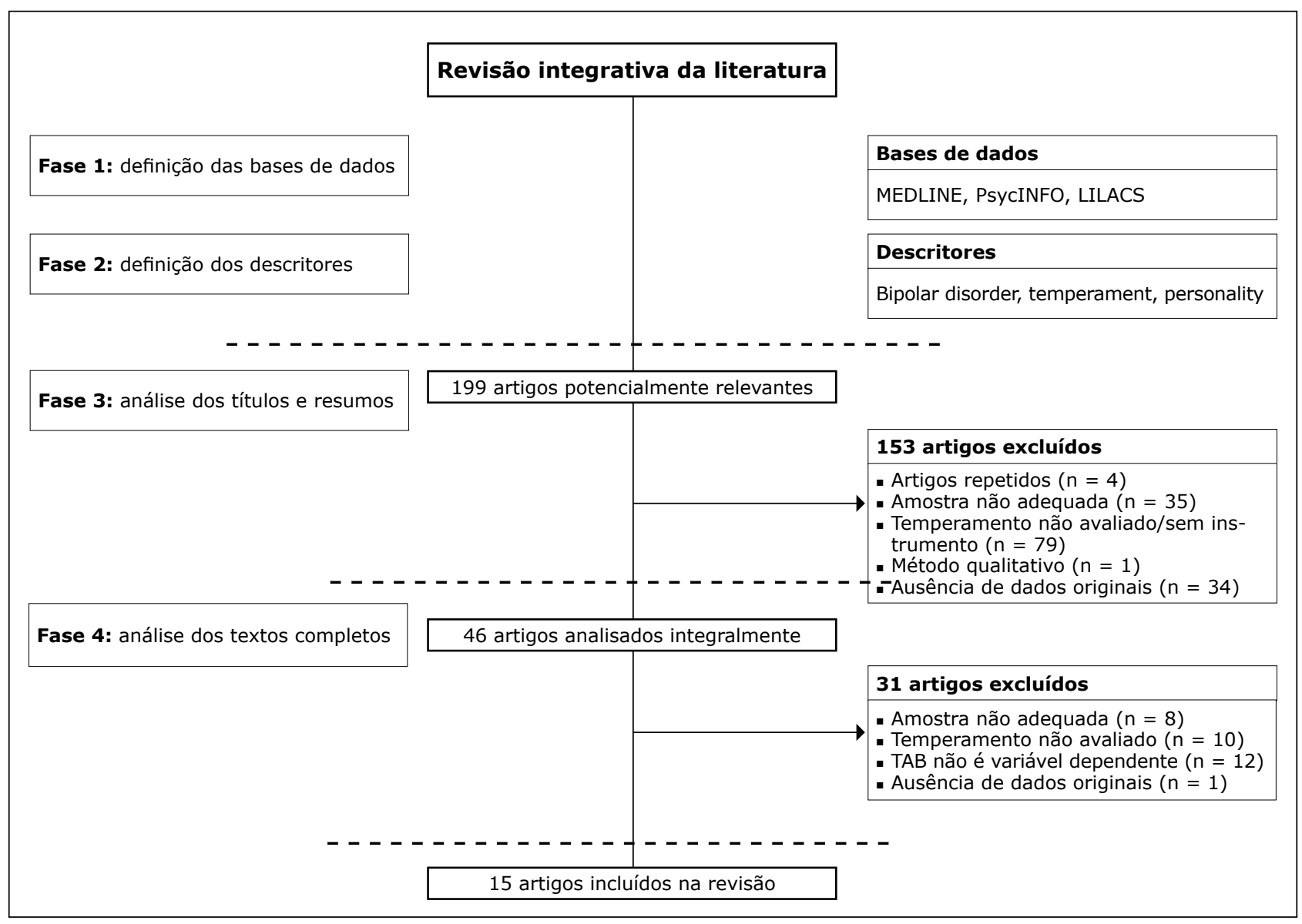

Figura 1 - Fluxograma do processo de revisão

\section{Populações clínicas estudadas}

As populações clínicas estudadas, de acordo com a faixa etária, foram as seguintes: crianças e adolescentes bipolares $(3 / 15)^{5,21,22}$, pacientes adultos bipolares ou do espectro bipolar (9/15) $)^{9,10,23-29}$ e crianças e adolescentes filhos de pais bipolares $(3 / 15)^{30-32}$. A inclusão desse último grupo se deu em função da hipótese de que essas crianças poderiam apresentar características temperamentais semelhantes àquelas diagnosticadas com o transtorno. Em geral, em todas as avaliações às quais as amostras clínicas foram submetidas, os participantes apresentavam estado de humor eutímico. Todos os estudos continham grupos controles formados por indivíduos sem histórico de diagnóstico de transtorno psiquiátrico.

\section{Instrumentos utilizados para o diagnóstico de sintomas de humor}

Em 14/15 estudos, os participantes dos grupos clínicos e controles foram selecionados por meio de entrevistas estruturadas realizadas por psiquiatras com base no Schedule for Affective Disorders and Schizophrenia (SADS), versão para crianças ou para adultos $5,21,22,25,26,30,32$.
O instrumento Structured Clinical Interview for DSM-IV (SCID) também foi utilizado isoladamente ou como fonte de informação complementar ao SADS em 6/15 estu$\operatorname{dos}^{9,23,24,27,29,30}$. Em 2/15 estudos, o Schedule for Clinical Assessment in Neuropsychiatry (SCAN) foi utilizado juntamente com o SCID ${ }^{29,30}$. Apenas em um estudo incluído na revisão não foi informado se o diagnóstico foi realizado com o auxílio de algum instrumento ${ }^{27}$.

Em relação à avaliação de sintomas maníacos e depressivos atuais no momento da coleta de dados, os seguintes instrumentos foram utilizados: Young Mania Rating Scale (YMRS) em 4/15,22,26,27; Hamilton Rating Scale for Depression (HAM-D) em 3/1522,26,27; e Inventário Beck de Depressão em 2/15 10,29. Finalmente, dentre os estudos, 5/15 utilizaram também outros instrumentos específicos, a saber, o Young Schema Questionnaire ${ }^{29}$, 0 Bech Rafaelsen Mania Scale e o Bech Rafaelsen Melancholia Scale ${ }^{28}$, o Longitudinal Interval Follow-up Evaluation (LIFE) ${ }^{25}$, o Positive and Negative Affect Scale $(\text { PANAS) })^{30}$ e o Altaman Self-Rating Mania Scale ${ }^{10}$. Esse levantamento indica que há uma variedade de instrumentos disponíveis para a avaliação de sintomas de humor, 
não havendo um consenso entre pesquisadores sobre o instrumento mais adequado para avaliar o estado de humor do paciente bipolar.

\section{Delineamento dos estudos}

Dentre os estudos selecionados, apenas 1/15 apresentou delineamento longitudinal ${ }^{28}$, sendo que os demais apresentaram delineamento transversal5,9,10,21-27,29,30,31,32.

É importante ressaltar que todos os estudos realizaram investigações comparativas e, por isso, não apresentaram análises da associação entre traços temperamentais e variáveis clínicas (tais como intensidade de sintomas de transtorno de humor, início e duração da doença, subtipo do primeiro episódio, comorbidades psiquiátricas e número de hospitalizações) ou outros aspectos psicopatológicos (tentativa de suicídio, grau de severidade da tentativa, histórico familiar de transtornos psiquiátricos, eventos estressores atuais e passados).

\section{Modelos teóricos e instrumentos para a avaliação dos traços temperamentais e principais resultados}

Nesta seção, para atender o objetivo desta revisão de literatura, optou-se por apresentar os artigos selecionados de acordo com a faixa etária da amostra utilizada, considerando que diferenças desenvolvimentais podem interferir na manifestação dos traços termperamentais. É importante observar que os estudos foram associados aos modelos a partir da referência explícita dos autores ou de acordo com o instrumento de avaliação de temperamento utilizado.

Amostras compostas por crianças e adolescentes

Entre os estudos avaliados, 6/15 de delineamento transversal utilizaram amostras compostas por crianças e adolescentes para investigar a relação entre características específicas de temperamento e aspectos clínicos dos transtornos de humor $5,21,22,30-32$.

É interessante notar que o desenvolvimento de dois desses estudos ${ }^{21,22}$ se fundamentou no modelo psicobiológico de temperamento e caráter de Cloninger et al. ${ }^{18}$, que investiga os traços de busca por novidades, esquiva ao dano e dependência da recompensa, e também aspectos do caráter, como persistência, autodirecionamento, cooperatividade e autotranscendência. Apesar de alguns resultados divergentes, esses estudos apontaram que crianças e adolescentes dos grupos clínicos apresentavam escores médios mais altos em busca por novidades, esquiva a danos e autotranscendência e escores médios mais baixos em persistência e autodirecionamento quando comparados a indivíduos saudáveis.

Em um terceiro artigo ${ }^{31}$, os autores avaliaram os traços de emocionalidade, atividade, sociabilidade e timidez, com base nas dimensões temperamentais propostas por Buss \& Plomin ${ }^{33}$. Os resultados indicaram que filhos de pais bipolares apresentavam elevação do traço emocionalidade. Já o estudo de Jones et al. ${ }^{30}$ foi baseado na teoria do reforçamento sensitivo de Jeffrey Gray (Behavioral Inhibition System and Behavioral Activation System Scales) ${ }^{19}$. Foram comparadas medidas de autorrelato de inibição e ativação comportamental e de humor entre crianças filhas de pais bipolares e crianças filhas de pais saudáveis. Diferentemente do que era esperado, não foram observadas diferenças significativas entre os escores médios das duas amostras.

Os dois artigos restantes ${ }^{5,32}$ avaliaram outras variáveis temperamentais que se aproximam conceitualmente das categorias delimitadas por Thomas \& Chess $^{34}$ em estudos com crianças pequenas, a saber: dificuldade de dormir, dificuldade de alimentação e maternagem, choro excessivo e de difícil consolo, cólicas, presença de estresse no momento de separação dos cuidadores e velocidade para apresentar afeto após a separação, capacidade de adaptação a novas situações, qualidade do humor e regularidade dos ritmos corporais. Essas variáveis temperamentais foram operacionalizadas em instrumentos específicos. De modo geral, os resultados dos estudos indicaram que crianças bipolares e filhas de pais bipolares tinham um estilo temperamental mais difícil nas dimensões avaliadas do que crianças saudáveis ${ }^{5,32}$.

A Tabela 1 apresenta uma síntese das principais características e resultados dos artigos selecionados para esta revisão e que utilizaram amostras compostas por crianças e adolescentes.

Nota-se dificuldade para realizar uma síntese dos resultados dos estudos selecionados, uma vez que diferentes modelos teóricos foram utilizados para conduzir a avaliação temperamental. De modo geral, pode-se afirmar que crianças e adolescentes com diagnóstico de TAB ou filhos de pacientes bipolares tendem a ser mais difíceis temperamentalmente do que crianças saudáveis. Essas crianças tendem a apresentar rotinas irregulares de sono e alimentação, choro relativamente frequente e alto, longos períodos para adaptação a novas rotinas, apesar de demonstrarem interesse por situações novas. A frustração produz tipicamente reações manhosas e violentas, o que pode estar associado à baixa persistência e ao baixo autodirecionamento.

Amostras compostas por indivíduos adultos

Amostras compostas por adultos foram avaliadas em

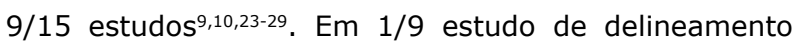
longitudinal, os traços de temperamento foram avaliados com base no modelo teórico dos três grandes fatores da personalidade propostos por Eysenck (neuroticismo, extroversão e psicoticismo) e também no fator desinibição ${ }^{28}$. Os resultados indicaram que altos escores no traço 
Traços de temperamento no transtorno bipolar - Vasconcelos et al.

Tabela 1 - Resumo dos estudos que investigaram traços de temperamento em pacientes com TAB: amostras de crianças e adolescentes, instrumentos utilizados na avaliação do temperamento e principais resultados

\begin{tabular}{lll}
\hline Estudos & Amostragem & Instrumentos \\
\hline Tillman et & Crianças e adolescentes bipolares & Inventário de Temperamento e \\
al. $(2003)^{21}$ & $(n=101)$, com TDAH $(n=68)$ & Caráter Júnior de Cloninger (JTCI), \\
& e crianças e adolescentes & versão para crianças e para os pais
\end{tabular}
saudáveis $(n=94)$

Resultados

Na percepção dos pais, crianças e adolescentes bipolares tendem a apresentar escores médios significativamente mais altos para busca por novidades e escores mais baixos para dependência de recompensa, persistência, autodirecionamento e cooperação.

A percepção de crianças e adolescentes a respeito de si próprios indicou que, quando comparadas ao grupo controle, elas tendem a apresentar escores mais altos para busca por novidades e escores mais baixos para persistência e autodirecionamento.

Jones et al. Adolescentes filhos de pais com $(2006)^{30} \quad$ TAB $(n=25)$ e crianças filhas de pais saudáveis $(n=22)$ com idade entre 13 e 19 anos

Duffy et al. Crianças e adolescentes filhos

$(2007)^{31}$ de pais portadores de TAB com e sem a manifestação de sintomas de humor ( $n=75)$ e crianças e adolescentes filhos de pais saudáveis $(n=30)$ com idade final média de 21 anos

West et al. Crianças com TAB $(n=25)$, $(2008)^{5} \quad$ com TDAH $(n=25)$ e indivíduos controles $(n=25)$ com idade média aproximada de 11 anos
Dysfunctional Attitudes Scale (DAS) Positive and Negative Affect Scale (PANAS) Behavioral Inhibition System and Behavioral Activation System Scales

Emotionality, Activity, Sociability and Shyness Temperament Questionnaire (EAS)

Não houve diferenças significativas nos escores médios das escalas de inibição/ativação comportamental, afeto positivo e na escala de atitudes disfuncionais entre as amostras de filhos de bipolares e filhos de adultos saudáveis.

Filhos de pais bipolares e que apresentavam sintomas psicopatológicos possuíam escores médios mais altos no traço emocionalidade quando comparados aos filhos de pais saudáveis.

Brief Scale of Temperament (BST)

Difficult Infant Behavior Scale (DIB)

Crianças bipolares apresentaram escores mais altos quando comparados a indivíduos controles em todas as dimensões temperamentais avaliadas: dificuldade de dormir, dificuldade de alimentação e maternagem, choro excessivo e de difícil consolo, cólicas, estresse no momento de separação dos cuidadores e velocidade para apresentar afeto após a separação, intensidade emocional. Crianças bipolares apresentaram escores mais baixos quando comparados a controles em: gosto por situações novas, capacidade de adaptação a novas situações (adaptabilidade), qualidade do humor e regularidade dos ritmos corporais.

Dimensions of Temperament-Revised (DOTS-R)

Filhos de pais bipolares apresentaram escores mais altos no nível de atividade geral e escores mais baixos na orientação para tarefa.
Singh et al. Crianças e adolescentes filhos

$(2008)^{32}$ de pais com TAB $(n=21)$ com idade entre 8 e 18 anos e crianças filhas de pais saudáveis $(n=31)$ que não apresentavam transtornos psiquiátricos do eixo I

Olvera Pacientes com TAB $(n=38)$ e et al. indivíduos controles $(n=31)$ $(2009)^{22} \quad$ com idade entre 8 e 17 anos
Inventário de Temperamento e Caráter Júnior de Cloninger (JTCI), versão para crianças e para os pais
Crianças e adolescentes diagnosticadas com TAB reportaram escores médios mais altos do que crianças controles nos aspectos busca por novidades, esquiva ao dano, autotranscendência. Crianças e adolescentes diagnosticados com TAB obtiveram escores médios mais baixos nos aspectos dependência de recompensa, persistência, autodirecionamento e cooperatividade. 
extroversão se associaram com o diagnóstico posterior de $T A B$, controlando as habilidades cognitivas.

Fora isso, 3/9 estudos compararam participantes saudáveis e indivíduos bipolares com relação a características de temperamento avaliadas no modelo de temperamento de Cloninger $9,26,27$. As dimensões de temperamento e caráter avaliadas foram explicitadas anteriormente ${ }^{18}$. A síntese dos principais resultados indicou que pacientes bipolares das três amostras tendiam a demonstrar escores mais baixos nos aspectos de caráter associados ao autodirecionamento e à cooperatividade quando comparados com indivíduos saudáveis ${ }^{9,26,27}$. Em dois estudos, foram observados escores significativamente mais altos nos pacientes bipolares nas dimensões busca por novidades, esquiva a danos e autotranscendência ${ }^{9,27}$.

Ainda, 5/9 avaliaram o temperamento de bipolares por meio da proposta teórica de Akiskal et al. ${ }^{13}$ e utilizaram a versão reduzida do instrumento de autorrelato desenvolvido pelo autor, denominado Temperament Evaluation of Memphis, Pisa, Paris and San Diego-Autoquestionnaire (TEMPS-A) ${ }^{9,10,23,24,29}$. No entanto, os resultados não foram consistentes, sendo que a comparação entre os grupos clínico e não-clínico em cada um dos estudos apresentou um perfil diferenciado. Três estudos apresentaram certa convergência em relação à elevação do traço ciclotimia na amostra de bipolares ${ }^{9,24,29}$, que demonstraram grande labilidade, com mudanças rápidas de humor, variação na quantidade de sono, energia, autoestima e socialização.

Um artigo de Akiskal et al. ${ }^{25}$ também foi incluído nessa revisão. Os autores utilizaram cinco medidas de traços temperamentais escolhidas a partir de sua relevância teórica para os transtornos de humor. Os 436 itens foram agrupados em quatro fatores a partir da análise fatorial exploratória, a saber: labilidade, energia-assertividade, sensibilidade (neuroticismo, insegurança, preocupação) e ansiedade social. Dessa forma, as diferenças encontradas entre os grupos foram significativas em todos os traços. Os bipolares tenderam a apresentar mudanças mais rápidas de humor, afirmar-se verbal ou fisicamente e ser mais instáveis emocionalmente quando comparados com o grupo de indivíduos sem transtornos de humor.

Finalmente, 1/9 estudo foi desenvolvido com base no modelo dos cinco grandes fatores da personalidade ${ }^{9}$. As variáveis de personalidade avaliadas foram: neuroticismo, extroversão, amabilidade, conscienciosidade e abertura a novas experiências ${ }^{16}$. Os resultados indicaram que os bipolares apresentam escores mais altos nas dimensões neuroticismo e abertura e apresentam escores mais baixos no traço conscienciosidade.

A Tabela 2 apresenta uma síntese das principais características e resultados dos artigos selecionados para esta revisão e que utilizaram amostras compostas por indivíduos adultos.

Tabela 2 - Resumo dos estudos que investigaram traços de temperamento em pacientes com TAB: amostras de adultos, instrumentos utilizados na avaliação do temperamento e principais resultados

\begin{tabular}{|c|c|c|c|}
\hline Estudos & Amostragem & Instrumentos & Resultados \\
\hline $\begin{array}{l}\text { Nowakowska } \\
\text { et al. }(2005)^{9}\end{array}$ & $\begin{array}{l}\text { Pacientes bipolares }(n=49) \text { e } \\
\text { controles saudáveis }(n=47)\end{array}$ & $\begin{array}{l}\text { Temperament Evaluation of } \\
\text { Memphis, Pisa, Paris and } \\
\text { San Diego-Autoquestionnaire } \\
\text { (TEMPS-A) } \\
\text { Neuroticism Extroversion } \\
\text { Openness Personality Inventory- } \\
\text { Revised (NEO-PI-R) } \\
\text { Inventário de Temperamento e } \\
\text { Caráter de Cloninger (TCI) }\end{array}$ & $\begin{array}{l}\text { Pacientes bipolares apresentaram escores } \\
\text { mais altos nos traços ciclotímico, irritável } \\
\text { e distímico da TEMPS-A. Nas dimensões } \\
\text { do NEO-PI-R, esses pacientes tiveram } \\
\text { escores mais altos nas dimensões } \\
\text { neuroticismo e abertura, e escores mais } \\
\text { baixos no traço conscienciosidade. Por } \\
\text { fim, no TCI, os bipolares apresentaram } \\
\text { escores mais altos no traço esquiva ao } \\
\text { dano, busca por novidades e na dimensão } \\
\text { autotranscendência, e escores mais baixos } \\
\text { na dimensão autodirecionamento. }\end{array}$ \\
\hline
\end{tabular}

Mendlowictz Pacientes bipolares $(n=23)$ e et al. $(2005)^{23}$ controles saudáveis $(n=102)$

Kesebir et al. $(2005)^{24}$
Temperament Evaluation of Memphis, Pisa, Paris and San Diego-Autoquestionnaire (TEMPS-A)

Temperament Evaluation of Memphis, Pisa, Paris and San Diego-Autoquestionnaire (TEMPS-A)
Não foram observadas diferenças significativas nas demais escalas. Bipolares apresentaram escores mais altos no traço ansioso e escores mais baixos no traço hipertímico.

Bipolares apresentaram escores mais altos nos traços ciclotímico e irritável, e escores mais baixos no traço depressivo. 
Tabela 2 - Resumo dos estudos que investigaram traços de temperamento em pacientes com TAB: amostras de adultos, instrumentos utilizados na avaliação do temperamento e principais resultados (cont.)

\begin{tabular}{lll}
\hline Estudos & Amostragem & Instrumentos \\
\hline Akiskal et al. & Pacientes bipolares tipo I $(\mathrm{n}=$ & Guilford-Zimmerman \\
$(2006)^{25}$ & $98)$, pacientes bipolares tipo II & Temperament Survey (GZT) \\
& $(\mathrm{n}=64)$, pacientes unipolares & Interpersonal Dependency \\
& com depressão maior $(\mathrm{n}=251)$ & Inventory (IDI) \\
& e indivíduos sem histórico de & Lazare-Klerman-Armor Personality \\
& diagnóstico $(\mathrm{n}=617)$ & Inventory (LZAP) \\
& & Maudsley Personality Inventory \\
& & (MPI)
\end{tabular}

Sayin et al. Pacientes com TAB eutímicos $(2007)^{26}$

\section{$(n=90)$ e grupo controle} formado por estudantes e pacientes psiquiátricos do eixo II $(n=90)$

Nery et al. $(2008)^{27}$ Pacientes com TAB ( $n=73)$ e indivíduos saudáveis $(n=63)$
Minnesota Multiphasic Personality Inventory (MMPI) Inventário de Temperamento e Caráter de Cloninger (TCI)

Inventário de Temperamento e Caráter de Cloninger (TCI)

Resultados

Pacientes bipolares apresentaram escores mais altos do que os indivíduos controles nas medidas de neuroticismo.

Pacientes com TAB no estado eutímico tiveram escores mais baixos nos fatores autodirecionamento e cooperatividade quando comparados com os controles saudáveis

Não houve associação entre estados de humor e os traços de temperamento avaliados pelo TCI.

Bipolares apresentaram escores mais altos em busca por novidades, esquiva a danos e autotranscendência, e baixos escores em autodirecionamento e cooperatividade. Bipolares sem comorbidades quando comparados a bipolares com comorbidades de transtornos ansiosos apresentaram escores médios mais baixos em busca por novidades e esquiva a danos. Por outro lado, apresentaram escores médios mais altos em dependência de recompensa, persistência, autodirecionamento, autotranscendência e cooperatividade.

Altos escores no traço extroversão mostraram associação com o diagnóstico posterior de $\mathrm{TAB}$, controlando as habilidades cognitivas.

Pacientes com transtornos afetivos apresentaram escores médios mais altos na dimensão temperamento depressivo quando comparados com indivíduos controles.

Pacientes bipolares apresentaram escores médios elevados na dimensão ciclotimia quando comparados com indivíduos controles.
Nilsson et al. $(2010)^{29}$
Indivíduos do sexo masculino, Defense Forces em 1982 ( $\mathrm{n}$ $=213.443$ ), sendo que 98 desenvolveram TAB

Pacientes com TAB tipo I ( $\mathrm{n}=$ 298), pacientes com TAB tipo II $(n=108)$, pacientes com depressão maior $(n=312)$ e indivíduos saudáveis $(n=209)$

Pacientes bipolares $(n=25)$, pacientes borderline $(n=31)$ e universitários saudáveis $(n=29)$
Big Three Personality - versão Multiphasic Personality Inventory (MMPI)

Temperament Evaluation of Memphis, Pisa, Paris and San Diego-Autoquestionnaire (TEMPS-A)

Temperament Evaluation of Memphis, Pisa, Paris and San Diego-Autoquestionnaire (TEMPS-A)

$\mathrm{TAB}=$ transtorno afetivo bipolar.

Novamente, nota-se dificuldade para realizar uma síntese dos resultados de estudos conduzidos com amostras de bipolares adultos devido à heterogeneidade dos modelos teóricos nos quais se baseiam. No entanto, é interessante que $1 / 15$ estudo tenha investigado a relação entre características de temperamento e predisposição para TAB com base em três das principais medidas utilizadas isoladamente nos demais estudos, a saber, Inventário de Personalidade Revisado (NEO-PI-R), TEMPS-A e Inventário de Temperamento e Caráter de Cloninger (TCI) ${ }^{9}$. $O$ estudo teve como objetivo geral comparar o perfil de personalidade de duas subamostras de transtornos afetivos (bipolar, depressão maior) com uma amostra de indivíduos saudáveis e de sujeitos criativos sem histórico 
de transtorno psiquiátrico. Os resultados indicaram que indivíduos bipolares, quando comparados com controles saudáveis, apresentaram escores significativamente mais altos nas dimensões ciclotimia, distimia e irritabilidade avaliadas por meio da TEMPS-A, nas medidas de neuroticismo obtidas por meio do NEO-PI-R e nos traços de esquiva a danos e busca por novidades do TCI. Por outro lado, observaram-se escores mais baixos para bipolares em conscienciosidade e autodirecionamento nos instrumentos NEO-PI-R e TCI, respectivamente.

A análise dos estudos quanto à sua classificação em termos de nível de evidência, com base nos critérios propostos por Manterola et al. ${ }^{20}$, indicou que poderiam ser classificados como estudos individuais de caso e controle (nível III).

\section{Discussão e conclusão}

O objetivo geral desta revisão foi identificar traços de temperamento que caracterizam pacientes bipolares em relação a indivíduos da população geral a partir de levantamento bibliográfico sistematizado. Os resultados dos 15 estudos revisados são difíceis de conciliar devido a importantes diferenças observadas nas características das amostras e nos diversos traços de temperamento investigados a partir de diferentes instrumentos e modelos teóricos.

Essa diversidade de resultados está relacionada à presença de diferentes concepções e dimensões a respeito do temperamento presentes nas teorias psicológicas desenvolvidas. Entre os estudos identificados, as teorias temperamentais propostas por Akiskal \& Akis$\mathrm{kal}^{35}$, Thomas \& Chess $^{34}$, Cloninger ${ }^{18}$ e Gray ${ }^{19}$ e a teoria dos cinco fatores ${ }^{9}$ foram utilizadas para fundamentar as investigações. Além disso, nota-se a ausência de modelos psicobiológicos consistentes teórica e empiricamente para explicar a natureza e o processo de desenvolvimento do temperamento normal e patológico. Consequentemente, as comparações carecem de força para permitir conclusões pertinentes a respeito da associação entre traços de temperamento e as variáveis relacionadas ao TAB. O estudo de Nowakowska et al. ${ }^{9}$ contribuiu parcialmente para delimitar o perfil geral de personalidade de pacientes bipolares, uma vez que a amostra foi avaliada por meio de diferentes modelos teóricos, conforme já descrito. No entanto, os autores optaram por caracterizar o perfil dos pacientes isoladamente em cada uma das escalas dos diversos instrumentos utilizados, mas não testaram a comunalidade entre elas, o que exigiria uma ampliação do tamanho amostral.

No intuito de delimitar um perfil geral de temperamento que caracterize pacientes bipolares, os resultados obtidos nos estudos desta revisão permitem afirmar que o perfil desse grupo tende a ser caracterizado por: a) rebaixamento dos traços avaliados no TCI relativos à persistência e ao autodirecionamento; b) elevação das medidas investigadas por meio do TCI referentes à busca por novidades, esquiva ao dano e autotranscendência; c) elevação das medidas relacionadas à dimensão neuroticismo tal como conceituada no modelo dos cinco grandes fatores $5,9,25,31,32$. A interpretação desses traços indica que os pacientes bipolares tendem a ser caracterizados como imaturos e dependentes, com dificuldade para se responsabilizar pelas consequências do próprio comportamento de acordo com suas escolhas, e tendem a abandonar tarefas diante de dificuldades. Ademais, tendem a evitar situações de punição, podendo apresentar-se como cuidadosos ou medrosos, pessimistas mesmo em situações em que a maioria das pessoas não demonstraria preocupação. Ao mesmo tempo, podem demonstrar curiosidade e entusiasmo por situações novas, porém são facilmente entediáveis. Tendem a ser impulsivos e desorganizados. Por fim, relatam ser despretensiosos, satisfeitos, pacientes, criativos, altruístas e espiritualizados. Em relação à instabilidade emocional, indivíduos com TAB tendem a experienciar emoções negativas, como preocupação, raiva, irritação, melancolia e vergonha. Características como tendência a ideias irracionais, baixo controle dos impulsos e dificuldade para enfrentar situações de estresse também compõem o perfil desses pacientes. A presença de características associadas ao neuroticismo em bipolares é convergente com os resultados de outros estudos, como o de Barnett et al. ${ }^{36}$. As implicações do perfil temperamental dos pacientes bipolares não foram discutidas em nenhum dos artigos analisados. No entanto, outros estudos presentes na literatura indicam que, dentre os desfechos psicopatológicos associados aos traços de temperamento no TAB, principalmente ao neuroticismo, estão a presença de ideação suicida ${ }^{37}$, comportamentos impulsivos ${ }^{38}$, entre outros.

Dentre as hipóteses sobre aspectos que interferem na consistência dos resultados observados, Akiskal et al. ${ }^{25}$ se referem à necessidade de trabalhar com amostras homogêneas quanto ao subtipo do TAB. Por exemplo, os autores discutem que a elevação das medidas de neuroticismo nos subtipos de transtornos de humor (bipolar tipo I, bipolar tipo II e unipolar) possui explicações diferentes. Eles sugerem que, em pacientes unipolares, o neuroticismo está associado a sintomatologia distímica subsindrômica; já em bipolares tipo II, esse traço temperamental estaria relacionado à labilidade afetiva habitual (ciclotimia) 25 . Entre os artigos analisados, apenas três identificaram separadamente bipolares tipo I e tipo $\mathrm{II}^{9,25,29}$, e diferenças temperamentais entre os dois grupos de pacientes foram encontradas. 
Observou-se também que alguns estudos inicialmente incluídos na revisão não foram mantidos, uma vez que os autores não foram atentos na definição constitutiva de traços de temperamento. Essa falha acarretou o uso de instrumentos não coerentes para avaliá-los, pois analisavam o estado de humor dos indivíduos, e não aspectos afetivos que tendem à estabilidade temporal. Consequentemente, as conclusões desses estudos eram pouco pertinentes para o objetivo desta revisão. Nesse sentido, nota-se também a importância de controlar o estado de humor do paciente no momento da avaliação da personalidade, para evitar a interferência da variação do humor nas respostas relacionadas a disposições e reatividade emocional ${ }^{25}$.

Outro aspecto referente ao ponto de vista teórico que pode ser ressaltado refere-se ao fato de que o desenvolvimento psicológico normal ou patológico depende das dimensões temperamentais, assim como da interação bidirecional entre elas e outras características psicológicas e demandas do ambiente ${ }^{39}$. As características temperamentais podem influenciar o tipo de interação que será estabelecida entre o indivíduo e seu ambiente, assim como o ambiente pode interferir nas oportunidades desenvolvimentais do próprio indivíduo. Dada a complexidade dessas inter-relações com os transtornos psiquiátricos, sua compreensão se apresenta como um processo trabalhoso e de longo prazo, uma vez que as relações não são necessariamente lineares. Nesse sentido, faz-se necessário desenvolver estudos longitudinais baseados em modelos multivariados que incluam variáveis individuais, clínicas e ambientais, considerando as interações entre elas. Os estudos longitudinais são relevantes à medida que permitem investigar variáveis preditoras para o desenvolvimento futuro de sintomas psiquiátricos ${ }^{40}$.

A dificuldade para estabelecer conclusões gerais com base nos resultados desta revisão pode estar relacionada a algumas limitações do próprio estudo. Do ponto de vista metodológico, nota-se que todos os estudos selecionados apresentaram delineamentos que caracterizam baixo nível de evidência. $O$ acesso a estudos longitudinais seria interessante no sentido de permitir identificar o perfil de temperamento pré-mórbido e, ainda, investigar se as dimensões de personalidade se mantêm constantes tanto em períodos eutímicos quanto em períodos de humor alterado, ou se refletem uma sintomatologia afetiva subsindrômica ${ }^{25}$. Os estudos longitudinais possuem ainda valor heurístico, pois contribuem para verificar a estabilidade dos traços de temperamento e suas relações com as variáveis clínicas dos transtornos psiquiátricos ${ }^{26}$. No entanto, nota-se uma carência na literatura de estudos longitudinais sobre o tema. Nesta revisão, foi identificado apenas um estudo longitudinal.

Outra limitação metodológica foi a seleção de descritores gerais para a busca dos estudos. A escolha justificou-se pela intenção de obter um número maior de estudos potenciais para o objetivo da revisão. No entanto, notou-se que os estudiosos tendem a utilizar como palavras-chave termos específicos relativos a traços de temperamento, o que impediu a identificação de outros estudos. É possível citar, a título de exemplo, os artigos publicados por Alloy et al. ${ }^{41}$, baseados no modelo da hipersensibilidade do BAS, nos quais as palavras-chave se circunscrevem ao nome do modelo teórico e/ou a um traço de temperamento em particular, e não incluem os termos temperamento ou personalidade. Revisões futuras devem ser mais específicas no sentido de incluir descritores relacionados a traços de temperamento de modelos teóricos particulares.

No entanto, apesar das limitações listadas, este estudo contribuiu para o levantamento do estado da arte da literatura sobre os traços de temperamento característicos de indivíduos com diagnóstico de TAB. Estudos recentes têm sugerido traços de temperamento como possíveis endofenótipos comportamentais do TAB e da depressão maior ${ }^{35,42}$. Gonda et al. ${ }^{43}$ identificaram uma associação significativa entre o alelo s do gene codificador do transportador de serotonina e os aspectos temperamentais que possuem um componente depressivo, tal como propostos por Akiskal. Em concordância com esse achado, no estudo desenvolvido por Gunthert et al. ${ }^{44}$, indivíduos com esse alelo tendiam a apresentar humor ansioso e depressivo. Os resultados dos trabalhos nessa linha de pesquisa ainda são controversos.

Diante das perspectivas da interação entre transtornos psiquiátricos e traços temperamentais e com base nos resultados desta revisão, enfatiza-se a importância de que, em estudos futuros, os construtos psicológicos básicos de interesse, a saber, temperamento e humor, sejam previamente delimitados. Esse aspecto pode ser útil para testar as possíveis combinações entre os construtos e, dessa forma, esclarecer a natureza dos prejuízos do funcionamento psicológico no TAB. Outro aspecto importante diz respeito à necessidade de escolher previamente o modelo teórico que conduzirá o desenvolvimento da investigação, considerando-se as várias propostas teóricas concorrentes. Além disso, o advento de técnicas estatísticas modernas, como equações estruturais, pode ser um caminho para os pesquisadores estudarem a estrutura latente dos transtornos mentais e características temperamentais, conforme os trabalhos de Krueger et al. ${ }^{45-47}$, que propuseram um modelo espectral que unifica os transtornos externalizantes e internalizantes e traços de personalidade por meio de um fator latente de ordem superior $^{45}$, que pode ser interpretado como a covariância (ou comorbidade) entre síndromes psicopatológicas e traços de personalidade. Essa perspectiva pode contribuir para o delineamento de estudos envolvendo o TAB e esclarecer, assim, a relação entre personalidade e sua etiopatogenia. 


\section{Referências}

1. Strelau J. Temperament: a psychological perspective. New York: Plenum; 1998.

2. Rothbart MK, Ahadi SA, Evans DE. Temperament and personality: origins and outcomes. J Pers Soc Psychol. $2000 ; 78(1): 122-35$.

3. Millon T, Lerner MJ, eds. Handbook of psychology. Hoboken: Wiley; 2003.

4. Flores-Mendoza C, Colom R. Introdução à psicologia das diferenças individuais. Porto Alegre: Artmed; 2006.

5. West AE, Schenkel LS, Pavuluri MN. Early childhood temperament in pediatric bipolar disorder and attention deficit hyperactivity disorder. J Clin Psychol. 2008;64(4):402-21.

6. Clark LA, Watson D, Mineka S. Temperament, personality and the mood and anxiety disorders. J Abnorm Psychol. 1994;103(1):103-16.

7. Caspi A, Henry B, McGee RO, Moffitt TE, Silva PA. Temperamental origins of child and adolescent behavior problems: from age three to age fifteen. Child Dev. 1995;66:55-68.

8. Krueger RF, Barlow DH, Watson D. Introduction to the special section: towards a dimensional based taxonomy of psychopathology. J Abnorm Psychol. 2005;114(4):492-3.

9. Nowakowska C, Strong CM, Santos CM, Wang PW, Ketter TA. Temperamental commonalities and differences in euthymic mood disorder patients, creative controls, and healthy controls. J Affect Disord. 2005;85:207-15.

10. Florio A, Hamshere M, Forty L, Green EK, Grozeva D, Jones $I$, et al. Affective temperaments across the bipolar-unipolar spectrum: examination of the TEMPS-A in 927 patients and controls. J Affect Disord. 2010;123:42-51.

11. Akiskal HS, Akiskal KK, Haykal RF, Manningd JS, Connor PD. TEMPS-A: progress towards validation of a self-rated clinical version of the Temperament Evaluation of the Memphis, Pisa, Paris, and San Diego Autoquestionnaire. J Affect Disord. 2005;85(1-2):3-16.

12. Erfurth A, Gerlach AL, Hellweg I, Boenigk I, Michael N, Akiskal HS. Studies on a German (Münster) version of the temperament auto-questionnaire TEMPS-A: construction and validation of the brief TEMPS-M. J Affect Disord. 2005;85:53-69.

13. Akiskal HS, Djenderedjian AM, Rosenthal RH, Khani MK. Cyclothymic disorder: validating criteria for inclusion in the bipolar affective group. Am J Psychiatry. 1977;134:1227-33.

14. Akiskal K, Akiskal HS. The theoretical underpinnings of affective temperaments: implications for evolutionary foundations of bipolarity and human nature. J Affect Disord. 2005;85:231-9.

15. Hirshfeld-Becker DR, Biederman J, Calltharp S, Rosenbaum ED, Faraone SV, Rosenbaum JF. Behavioral inhibition and disinhibition as hypothesized precursors to psychopathology: implications for pediatric bipolar disorder. Biol Psychiatry. 2003;53:985-99.

16. McCrae RR, Costa PT. Validation of the five-factor model of personality across instruments and observers. J Pers Soc Psychol. 1987;52(1):81-90.

17. Caspi A, Silva PA. Temperamental qualities at age 3 predict personality traits in young adulthood: longitudinal evidence from a birth cohort. Child Dev. 1995;66:486-98.

18. Cloninger CR, Svrakic DM, Przybeck TR. A psychobiological model of temperament and character. Arch Gen Psychiatry. 1993;50:975-90.

19. Alloy LB, Abramson LY, Walshaw PD, Cogswell A, Grandin LD, Hughes ME, et al. Behavioral approach system and behavioral inhibition system sensitivities and bipolar spectrum disorders: prospective prediction of bipolar mood episodes. Bipolar Disord. 2008;10:310-22.

20. Manterola CD, Pineda VN, Vial MG, Losada HM, Muñoz SN. Revisión sistemática de la literatura. Propuesta metodológica para su realización. Rev Chilena Cir. 2003;55(2):204-8.

21. Tillman R, Geller B, Craney JL, Bolhofner K, Williams M, Zimerman $B$, et al. Temperament and character factors in a prepubertal and early adolescent bipolar disorder phenotype compared to attention deficit hyperactive and normal controls. J Child Adolesc Psychopharmacol. 2003;13(4):531-43.

22. Olvera RL, Fonseca M, Caetano SC, Hatch JP, Hunter K, Nicoletti $M$, et al. Assessment of personality dimensions in children and adolescents with bipolar disorder using the Junior Temperament and Character Inventory. J Child Adolesc Psychopharmacol. 2009;19(1):13-21.

23. Mendlowicz MV, Jean-Louis G, Kelsoe JR, Akiskal HS. A comparison of recovered bipolar patients, healthy relatives of bipolar probands, and normal controls using the short TEMPS-A. J Affect Disord. 2005;85:147-51.

24. Kesebir S, Vahip S, Akdeniz F, Yuncu Z, Alkan M, Akiskal HS. Affective temperaments as measured by TEMPS-A in patients with bipolar I disorder and their first-degree relatives: a controlled study. J Affect Disord. 2005;85:127-33.

25. Akiskal HS, Kilzieh N, Maser JD, Clayton PJ, Schettler PJ, Shea $\mathrm{T}$, et al. The distinct temperament profiles of bipolar I, bipolar II and unipolar patients. J Affect Disord. 2006;92:19-33.

26. Sayin A, Kuruoglu AÇ, Güleç MY, Aslan S. Relation of temperament and character properties with clinical presentation of bipolar disorder. Compr Psychiatry. 2007;48(5):446-51.

27. Nery FG, Hatch JP, Glahn DC, Nicoletti MA, Monkul ES, Najt $P$, et al. Temperament and character traits in patients with bipolar disorder and associations with comorbid alcoholism or anxiety disorders. J Psychiatr Res. 2008;42(7):569-77.

28. Lönnqvist JE, Verkasalo M, Haukka J, Nyman K, Tiihonen J, Laaksonen I, et al. Premorbid personality factors in schizophrenia and bipolar disorder: results from a large cohort study of male conscripts. J Abnorm Psychol. 2009;118(2):418-23.

29. Nilsson AKK, Jørgensen CR, Straarup KN, Licht RW. Severity of affective temperament and maladaptive self-schemas differentiate borderline patients, bipolar patients, and controls. Compr Psychiatry. 2010;51:486-91.

30. Jones SH, Tai S, Evershed K, Knowles R, Bentall R. Early detection of bipolar disorder: a pilot familial high-risk study of parents with bipolar disorder and their adolescent children. Bipolar Disord. 2006;8(4):362-72.

31. Duffy A, Alda M, Trinneer A, Demidenko N, Grof P, Goodyer IM. Temperament, life events, and psychopathology among the offspring of bipolar parents. Eur Child Adolesc Psychiatry. 2007; 16:222-8.

32. Singh MK, DelBello M, Strakowski SM. Temperament in child offspring of parents with bipolar disorder. J Child Adolesc Psychopharmacol. 2008;18(6):589-93.

33. Buss AM, Plomin R. Temperament: early developing personality traits. Hillsdale: Lawrence Erlbaum; 1984.

34. Thomas A, Chess S. Temperament and development. New York: Brunner/Mazel; 1977.

35. Akiskal HS, Akiskal K. Cyclothymic, hyperthymic and depressive temperaments as subaffective variants of mood disorders. In: Tasman A, Riba MB, eds. Annual review of psychiatry. Washington: American Psychiatric Press; 1992. p. 43-62.

36. Barnett JH, Huang J, Perlis RH, Young MM, Rosenbaum JF, Nierenberg AA, et al. Personality and bipolar disorder: 
dissecting state and trait associations between mood and personality. Psychol Med. 2010;7:1-12.

37. McCann SJH. Suicide, big five personality factors, and depression at the American state level. Arch Suicide Res. 2010;14(4):368-74.

38. Lewis M, Scott J, Frangou S. Impulsivity, personality and bipolar disorder. Eur Psychiatry. 2009;24:464-9.

39. Ito PCP, Guzzo RSL. Temperamento: características e determinação genética. Psicol Reflex Crit. 2002;15(2):425-36.

40. Morgan GA, Gliner JA, Harmon R. Clinicians' guide to research methods and statistics. J Am Acad Child Adolesc Psychiatry. 1999;38(12):1595-7.

41. Alloy LB, Abramson LY, Walshaw PD, Cogswell A, Smith JM, Neeren AM, et al. Behavioral Approach System (BAS) sensitivity and bipolar spectrum disorders: a retrospective and concurrent behavioral high-risk design. Motiv Emot. 2006;30:143-55.

42. Azorin JM, Kaladjian A, Adida M, Hantouche EG, Hameg A, Lancrenon S, et al. Psychopathological correlates of lifetime anxiety comorbidity in bipolar I patients: findings from a French national cohort. Psychopathology. 2009;42:380-6.

43. Gonda X, Rihmer Z, Zsombak T, Bagdy G, Akiskal K, Akiskal HS. The 5-HTTLPR polymorphism of the serotonin tra nsporter gene is associated with affective temperaments as measured by TEMPS-A. J Affect Disord. 2006;91:125-31.
44. Gunthert KC, Conner TS, Armeli S, Tennen H, Covault J, Krangler HR. Serotonin transporter gene polymorphism (5-HTTLPR) and anxiety reactivity in daily life: a daily process approach to gene-environment interaction. Psychosom Med. 2007;69:762-8.

45. Krueger RF, Hicks BM, Patrick CJ, Carlson SR, Iacono WG, McGue M. Etiologic connections among substance dependence, antisocial behavior, and personality: modeling the externalizing spectrum. J Abnorm Psychol. 2002; 107:216-27.

46. Krueger RF. The structure of common mental disorders. Arch Gen Psychiatry. 1999;56:921-6.

47. Krueger RF, Caspi A, Moffit T, Silva PA. The structure and stability of common mental disorders (DSM-III-R): a longitudinal-epidemiological study. J Abnorm Psychol. $1998 ; 107(2): 216-27$.

\section{Correspondência}

Alina Gomide Vasconcelos

Universidade Federal de Minas Gerais, Faculdade de Medicina Av. Alfredo Balena, 190, sala 325 - Santa Efigênia

30130-100 - Belo Horizonte, MG - Brasil

E-mail: alinagomide@gmail.com 